American Journal of Infectious Diseases 4 (1): 1-9, 2008

ISSN 1553-6203

(C) 2008 Science Publications

\title{
Immunopathogenesis of Dengue Hemorrhagic Fever
}

\author{
${ }^{1}$ Huan-Yao Lei, ${ }^{1}$ Kao-Jean Huang,, Yee-Shin Lin, ${ }^{2}$ Trai-Ming Yeh,,${ }^{1}$ Hsiao-Sheng Liu and ${ }^{3}$ Ching-Chuan Liu \\ Departments of Microbiology and Immunology, ${ }^{2}$ Medical Technology and ${ }^{3}$ Pediatrics, \\ College of Medicine, National Cheng Kung University, Tainan, Taiwan
}

\begin{abstract}
Dengue virus infection causes Dengue Fever (DF), Dengue Hemorrhagic Fever (DHF) and Dengue Shock Syndrome (DSS) whose pathogeneses were not clearly understood. A new hypothesis of immunopathogenesis is proposed for the development of the DHF/DSS. An aberrant immune overactivation after dengue virus infection not only impair the immune response to clear the virus, but also result in overproduction of cytokines that affect monocytes, endothelial cells, and hepatocytes, as well as the abnormal production of autoantibodies to platelet and endothelial cells. A molecular mimicry occurs between platelets/endothelial cells and dengue virus antigens. Platelets and endothelial cells are bound by the cross-reactive anti-dengue virus antibodies such as anti-NS1 or anti-prM antibodies. The IFN- $\gamma$ activated macrophage would phagocytosize the osponized targets. Dengue virus-induced vasculopathy and coagulopathy are involved in the pathogenesis of hemorrhage, and the unbalance between coagulation and fibrinolysis activation increases the likelihood of severe hemorrhage in DHF/DSS. This theory of transient hemophagocytic activity in immunopathogenesis of DHF/DSS can account for specific characteristics of clinical, pathologic, and epidemiological observations in dengue virus infection.
\end{abstract}

Key words: Dengue virus, ADE, autoantibody, macrophage

\section{INTRODUCTION}

Dengue fever is an acute infectious disease caused by four serotypes of dengue virus. It is characterized by biphasic fever, myalgia, headache, pain in various parts of the body, rash, lymphadenopathy, and leucopenia ${ }^{[1,2]}$. DF is self-limited, but, it will progress to Dengue Hemorrhagic Fever (DHF) or Dengue Shock Syndrome (DSS) in certain conditions. DHF is a severe febrile disease characterized by abnormalities of hemostasis and increased vascular permeability, and severe progression may result in DSS. DSS is a form of hypovolemic shock that is associated clinically with hemoconcentration and which might lead to death if appropriate care is not given. After dengue virus infection, there is a continuum from mild DF to severe DHF or DSS. It has been estimated that only $4-6 \%$ of individuals with secondary infection develop severe DHF disease ${ }^{[3,4]}$. Dengue virus can infect infant, children and adult. In endemic area such as southeastern Asia or Latin American, most of the DHF/DSS are children while some are infants. However, in non-endemic area like Taiwan, the majority of the DHF/DSS case is adult or the elder. The dengue-infected elder will have more severe clinical symptoms, sometime is fatal $^{[5]}$. The pathogenesis, especially the mechanistic steps toward the manifestation of DHF/DSS, involved in this process is not clearly understood. Any explanation of the DHF/DSS pathogenesis must account for specific characteristics of clinical, pathologic, and epidemiological observations that are unique in dengue virus induced disease.

Hypotheses on the pathogenesis of dengue hemorrhagic fever: Several hypotheses for the pathogenesis of dengue hemorrhagic fever have been proposed. Among them, Antibody-Dependent Enhancement (ADE) of infection has long been thought to play a central role ${ }^{[6,7]}$. The ADE hypothesis was formulated to explain the finding that severe manifestations of DHF/DSS occur in children experiencing a second dengue virus infection that has a different serotype from previous one. There are indeed preexisting antibodies to previous dengue virus that cannot neutralize but rather enhance infection in vitro. Sera obtained before infection from children who later developed DHF/DSS were much more likely to demonstrate ADE in vitro than those who had only $\mathrm{DF}^{[8]}$. Newborn babies less than 1 year old who acquire

Corresponding Author: Dr. Huan-Yao Lei, Department of Microbiology and Immunology, College of Medicine, National Cheng Kung University, Tainan 701, Taiwan, Republic of China 
maternal anti-dengue IgG antibody are also susceptible to developing DHF/DSS post primary infection ${ }^{[9]}$. Epidemiological studies support the association of DHF/DSS with secondary dengue virus infection. However, the association of DHF/DSS with prior immunity to other dengue serotypes by itself explains neither the pathogenetic basis of the association nor the molecular mechanism of DHF/DSS clinical manifestations. It is not clear how augmentation of dengue virus infection by enhancing antibodies leads to DHF/DSS.

Virus virulence, the capacity of a virus to produce disease in a host, is an alternative hypothesis for the pathogenesis of DHF/DSS. The different manifestations of DF, DHF, and DSS may be caused by variants of dengue virus with different degree of virulence. The risk of DHF/DSS is higher in secondary infections with dengue virus of serotype 2 compared with to that the other serotypes ${ }^{[10,11]}$. Structural differences have also been found among various isolates of DF and DHF patients $^{[12]}$. Furthermore, it was reported that high dengue viremia titer was associated with increased disease severity ${ }^{[13,14]}$. Peak viral titers were 10 - to 100 fold higher in patients with DSS than those with DF in dengue-infected Thai children. Viral load is a contributing factor in the development of DHF/DSS. But, the virus load has dynamic change, varying from individual to individual, or even from day to day post infection. It declines quickly at the day of fever subsided. The viral load is reflective of its virulence, the high growth rate in vivo, or the consequence of the host immune response needs further differential investigation. However, persons infected with the same dengue virus will have different clinical manifestations, suggesting that host factors must play important roles in the development of the dengue disease.

Immunopathogenesis in dengue hemorrhagic fever has been proposed ${ }^{[15,16]}$. Serotype cross-reactive antibodies from the previous infection bind to virions without neutralization and enhance the entry of virus into monocytes. The number of virus-infected monocytes increases. As a result, the level of dengue virus-specific $\mathrm{T}$ cell activation is markedly enhanced. The $\mathrm{T}$ cells, especially the cross-reactive $\mathrm{T}$ cells, produce cytokines such as IFN- $\gamma$, IL-2 and TNF $\alpha$ and lyse dengue virus-infected monocytes. TNF $\alpha$ is also produced by activated monocytes. The complement cascade is activated by a virus-antibody complex as well as by several cytokines to release $\mathrm{C} 3 \mathrm{a}$ and $\mathrm{C} 5 \mathrm{a}$ which also have direct effects on vascular permeability. The synergistic effects of IFN- $\gamma$, TNF $\alpha$ and activated complement proteins trigger plasma leakage of endothelial cells in secondary dengue virus infection.
However, several issues remain unclear by this theory. Not all DHF/DSS cases are secondary infections. Although most of the DHF/DSS in children are secondary infection, but the DHF/DSS in infants are primary infection. Complement activation may be the result of severe disease, not the cause of DHF/DSS. DHF develops rapidly, usually over a period of hours, and resolves within 1 to 2 days in patients who receive appropriate fluid resuscitation. It is also not clear why both endothelial cells and platelets are the major targets to affect in dengue disease. No discernible sequelae are usually found. This scenario is not easily reconciled with the known tissue-destructive effects of inflammatory cytokines.

We have proposed an alternative immunopathogenesis for dengue virus infection ${ }^{[17]}$. Dengue virus infection causes aberrant immune responses. These aberrant immune responses not only impair the immune response to clear the virus, result in overproduction of cytokines, as well as abnormal production of autoantibodies. The aberrant generation of anti-NS1 antibodies that cross-react with platelet or endothelial cells initiates the subsequent development of dengue disease. Anti-platelet or anti-endothelial cell autoantibodies must be involved in the clinical manifestation of thrombocytopenia and endothelial cell dysfunction. Dengue virus infection-caused endothelial damage and bleeding also contribute to the hemorrhage, and the imbalance between coagulation and fibrinolysis activation increases the likelihood of severe hemorrhage in DHF/DSS. Hemostasis is maintained unless the dysregulation of coagulation and fibrinolysis persists. The dengue virus induced autoantibodies and pathogenetic changes will be discussed in the following sections.

Clinical and pathologic manifestation of dengue virus infection: Dengue fever is an acute febrile illness with headache, retro-orbital pain, myalgia, arthralgia, rash, leukopenia and mild thrombocytopenia. Biphasic fever and rash are the most characteristic features of classic dengue fever. Symptoms resolve after 2 to 7 days. Dengue hemorrhagic fever is an acute vascular permeability syndrome accompanied by abnormalities in hemostasis. The clinical features include plasma leakage, bleeding tendency, and liver involvement ${ }^{[1,2]}$. Capillary leakage develops rapidly over a period of hours, near or at the end of the febrile period when the symptoms of classic DF resolve. Pleural effusion, ascites, and hemoconcentration are indicative of intravascular volume loss. It can quickly progress to shock if patients do not receive intravascular fluid resuscitation. The hemorrhagic manifestations range 
from a positive tourniquet test to spontaneous bleeding from the nose or the gastrointestinal tract. Hemoconcentration and marked thrombocytopenia are two major characteristic features of DHF/DSS. Liver involvement is common in dengue virus infection with mild elevation of serum transaminases. Ample studies demonstrate that dengue virus can infect a variety of human primary cells including monocytes/macrophages, dendritic cells, B cells, hepatocytes, Kupffer cells and cell lines of endothelial and epithelial origin. However, these in vitro infections are modulated by the cell type and viral $\operatorname{strain}^{[18]}$. Therefore, damage or dysfunction of these cells or organs induced by dengue virus infection, either directly or indirectly is responsible for the progressive development of DHF/DSS.

Dengue virus-induced aberrant immune activation: We have reported that an abnormal immune status in dengue patients during a dengue serotype 3 Taiwan outbreak in $1998^{[19]}$. The immunophenotypes of consecutive blood samples from 29 dengue patients, of whom 21 had DF and 8 had DHF/DSS were determined by flow cytometry and fond that early activation marker CD69 appeared on lymphocytes and monocytes at day 4 post fever onset, and declined afterward. However, a transient reverse in the CD4/CD8 ratio occurred at day 6 to 14 post fever onset. The CD4/CD8 ratio inversion was manifested in 10 out of 29 dengue patients, and it was more frequently encountered in DHF/DSS than in DF patients. Analysis of the clinical blood cell count of these 10 cases showed that increase of immature neutrophils developed at fever day 5-6, CD $4{ }^{\mathrm{dim}}$ or CD8 ${ }^{\text {dim }}$ monocytosis at day 6-7 and atypical lymphocytosis at day 8-10 post fever onset. The PHAstimulated $\mathrm{T}$ cell response was depressed as well. These changes in immune parameters are indicative of aberrant immune activation during dengue virus infection and might be involved in the initiation of the dengue pathogenesis.

Dengue virus can infect immature dendritic cells, monocytes, as well as B cells. Monocytes were the major target for dengue virus infection, but they will undergo apoptosis to prevent the spread of virion, but also decrease the virus-induced inflammation because the apoptotic cells will be phagocytosed by macrophages. Although the immature dendritic cells were reported to be 10-times more permissive for dengue virus infection than monocytes or macrophages ${ }^{[20]}$, but no antibody enhancement of infection was observed even though they express Fc receptors. The infection of dendritic cells stimulates their maturation and cytokine production of TNF $\alpha$ and
IFN $\alpha$, but not of IL- 6 and IL-12 $2^{[21]}$. The levels of IL-12 are higher in DF than DHF patients. No IL-12 could be detected in patients with DHF grades III and $\mathrm{IV}^{[22]}$. This deficiency in IL-12 production might lead to a shift to the Th2-type response and inappropriate CTL generation. In addition to monocytes or dendritic cells, human B cells can also support dengue virus replication in vitro. The dengue virion production, as well as enhancing antibody phenomenon on dengue virion and TNF- $\alpha$ /IL- 6 cytokine release were similar in both B cells and monocytes ${ }^{[23]}$. King et al., have reported that B cells were the principal circulating mononuclear cells infected by dengue virus. The majority of the virus was recovered from $\mathrm{CD} 20^{+} \mathrm{B}$ cells of dengue patients ${ }^{[24]}$. The effect of dengue infection on $B$ cells, together with the overproduction of B-cell growth factor IL-6, might trigger the maturation of plasma cells and the generation of autoantibodies that will be discussed later. These immune deviations not only delay virus clearance, but also trigger cytokine overproduction and auto-anti-platelet antibodies that initiate the subsequent pathogenesis of dengue virus infection.

Since mononuclear cells are overactivated during acute dengue infection, it is expected that elevated levels of cytokines can be found in serum. Indeed, there are elevations of serum cytokine levels in dengue patients. High levels of T-cell activation markers such as the soluble IL-2 receptor, soluble CD4, soluble CD8, IL-2, and IFN- $\gamma$, as well as monokines, e.g., TNF $\alpha$, IFN- $\beta$ and GM-CSF, were detected in dengue-infected children, and these markers were higher in DHF/DSS patients than in DF patients ${ }^{[25,26]}$. High serum levels of inhibitory cytokines such as IL-10 or the soluble receptors of sTNFRI and sTNFRII were also found in $\mathrm{DHF}^{[27]}$. We also reported a mixed Th1/Th2 cytokine profile in primary infection of infant DHF and secondary infection of children $\mathrm{DHF}^{[28]}$. Both proinflammatory cytokines (IFN- $\gamma, \mathrm{TNF}-\alpha$ ) and antiinflammatory cytokines (IL-10, IL-6) were elevated in the sera of infants and children with DHF/DSS. However, the concentrations of IFN- $\gamma$ and IL-10 were not different between children with secondary dengue infections and infants with primary dengue infections. No increase of IL-4 and IL-2 were found. This is different from the Rothman and Ennis's viewpoint that cross-reactive $\mathrm{T}$ cells in response to secondary infection of different serotype can produce more cytokines and cause more severe $\mathrm{DHF}^{[29]}$. It seems that enhancing antibodies from either mothers (primary infection in infants) or previous infection (secondary infection in children) will promote infection of Fc-bearing cells resulting in a large infected cell mass in patients. The $\mathrm{T}$ 
cell and cytokine responses are proportional to the infected cell mass. The inflammatory cytokines of IFN- $\gamma$ and anti-inflammatory cytokines of IL-10 are present simultaneously in DHF/DSS patients. The host responds to dengue virus infection via generation of inflammatory cytokines. Simultaneously, the inhibitory cytokines were also induced to counteract the inflammation. Cytokines can cause cell activation synergistically or antagonistically; the net outcome will depend on the balance between various cytokine actions.

Dengue-infected patients usually are leukopenic for several days during the acute infection, characterized by a decrease in the absolute number of neutrophils and monocytes. However, there are transient neutrophila and monocytosis before leukopenic ${ }^{[19]}$. Dengue virus stimulation induced neutrophils activation to release chemokines such as IL-8, MIP- $1 \alpha$ and MIP-1 $\beta$, as well as neutrophil granular enzyme, MPO and to express CD11b/CD18 and Toll-like receptor 4. The neutrophil or monocyte activation also contributes to the pathogenesis of DHF/DSS.

Dengue virus-induced anti-platelet and antiendothelial cell autoantibodies: Thrombocytopenia is common in dengue fever and always found in DHF/DSS ${ }^{[1,2]}$. The pathogenesis of thrombocytopenia is poorly understood. It was suggested that dengue virusinduced bone marrow suppression depressed platelet synthesis and resulted in thrombocytopenia ${ }^{[30]}$. The dengue- 2 virus can also bind to human platelets in the presence of virus-specific antibodies, and an immunemediated clearance of platelets was proposed to involve in the pathogenesis of thrombocytopenia in DHF/DSS ${ }^{[31]}$. Parvovirus infection is reported to associate with childhood idiopathic thrombocytopenic purpura $^{[32,33]}$. We have reported that $\operatorname{IgM}$ anti-platelet autoantibodies in dengue patients or in dengue virusinfected mice ${ }^{[34,35]}$. The titer of $\operatorname{IgM}$ anti-platelet antibodies is higher in DHF/DSS than in DF patients. The presence of these autoantibodies not only induces platelet lysis via complement activation, but also inhibits ADP-induced platelet aggregation. The antiplatelet antibody binding activity was not observed in other virus-infected patient sera. Although there was no difference or correlation between the anti-platelet IgM levels with the platelet counts both in infants and children with DHF/DSS, or non-shock DHF versus DSS, auto anti-platelet antibodies can indeed contribute to the destruction of platelets ${ }^{[36]}$.

The binding reactivity of the sera of DHF/DSS with endothelial cells was detected by flow cytometric analysis. There were higher percentages of endothelial cells reactive with DHF/DSS than those with DF patient sera $^{[37]}$. There were both IgM and IgG anti-endothelial cell antibodies. The levels of anti-endothelial cell antibodies were not different between non-shock DHF and DSS in infants or children. No relation between the levels of anti-endothelial cell autoantibodies and the increase in hematocrit, an evidence of plasma leakage was observed. Furthermore the anti-platelet and antiendothelial cell IgM or IgG levels were not different between primary infection of infant and secondary infection of children. Further studies showed that the platelet or endothelial cell binding activities were inhibited by pretreatment with dengue virus nonstructural protein 1 (NS1). A molecular mimicry between the dengue virus and endogenous self-proteins was proposed to be one of the mechanisms for the induction of autoimmunity during dengue virus infection.

Dengue virus-induced vasculopathy: The most characteristic feature of DHF/DSS and the best indicator of disease severity is plasma leakage. Plasma leakage is caused by a diffuse increase in capillary permeability and manifests as any combination of hemoconcentration, pleural effusion, or ascites. It usually becomes evident on days 3-7 of illness, during which time dengue fever resolve ${ }^{[1,2]}$. Plasma leakage occurs systemically, progressing quickly, but will resolve within 1 to 2 days in patients who receive appropriate fluid resuscitation. No subsequent tissue or organ dysfunction is observed. Although perivascular edema is obvious, no obvious destruction of vascular endothelial cells has been reported. It was previously thought that plasma leakage was due to altered vascular permeability rather than to structural destruction of endothelial cells. The functional alteration of endothelial cells is probably caused via by-standard effects of cytokine or mediator release in dengue infection. The dengue virus can infect endothelial cells in vitro which leads to apoptosis as well as production of cytokines and chemokines such as IL-6, IL-8 and RANTES $^{[38,39]}$, but no infection on human DHF/DSS biopsy has been demonstrated.

We have reported that the anti-NS1 antibodies can cross-react with non-infected endothelial cells and induce these cells to undergo apoptosis ${ }^{[40]}$. The signaling pathway involved the expression of iNOS and the production of NO after stimulation by anti-NS1 antibodies $^{[42]}$. Production of NO further caused upregulation of $\mathrm{p} 53$ and $\mathrm{Bax}$ and down-regulation of Bcl-2 and Bcl-xL, the pro- and anti-apoptotic factors that lead to cytochrome c release and caspase-3 
activation. Tyrosine phosphorylation and NF- $\kappa B$ activation were found after anti-NS1 stimulation in endothelial cells ${ }^{[40]}$. Furthermore, the anti-prM antibody can bind to endothelial cells, one of the antigens is heat shock protein (HSP) $60^{[43]}$.

Dengue virus-infected endothelial cells are capable of activating complement and inducing the expression of adhesion molecules such as ICAM-1 ${ }^{[44]}$. The expression of ICAM-1 together with the production of chemokines IL-8 and RANTES increases the adherence of polymorphonuclear cells and mononuclear cells, respectively, and results in increased vasopermeability and thrombomodulin release, a marker of endothelial cell damage. Serum levels of TM in infants and children with DHF/DSS were higher than those of controls, but their levels were not correlated with the severity of DHF in infants and children. Although there was no correlation between the serum levels of TM and the levels of anti-endothelial cell autoantibodies as well as the increase in hematocrit in infants and children with DHF/DSS, this indicates that endothelial cell structural damage occurred in vivo under certain conditions. It seems that both direct viral cytopathic effects and immune mediated damage by leukocyte recruitment can cause structural injury to infected endothelial cells. This vascular leakage can be induced either directly or indirectly during dengue virus infection. Moreover, although the endothelial cell surface molecules that are recognized by these autoreactive antibodies need to be identified, a crossreactivity exists between endothelial cells and dengue virus antigens due to the molecular mimicry. The endothelial cells do not need to be infected by dengue virus to be targeted. The cross-reactive anti-dengue antibody such as anti-NS1 or anti-prM can bind to the un-infected endothelial cells to cause its damage. Because endothelium plays a crucial role in maintaining hemostasis, damage of endothelial cells during dengue virus infection may skew the procoagulant/ anticoagulant balance of endothelium and increase the bleeding tendency. The sequestration of platelets by activated endothelial cells might also contribute to the development of thrombocytopenia.

Dengue virus-induced coagulopathy: Some viral infections can cause hemostatic abnormalities. Hemorrhage is a consequence of either a more pronounced degree of thrombocytopenia and associated platelet dysfunction or disseminated intravascular coagulation. In dengue virus-induced hemorrhagic manifestations, platelet-vascular abnormalities are more common, but as the severity of illness progresses, massive bleeding with disseminated intravascular coagulation can occur. Hemostasis is maintained by the balance between coagulation and fibrinolysis. The coagulation system can be activated by both intrinsic and extrinsic pathways to form thrombin which converts fibrinogen to fibrin. The fibrinolytic system, on the other hand, can break down fibrin into fibrin degradation products. The human fibrinolytic system is comprised of plasminogen, a proenzyme, which can be activated to the active plasmin enzyme by several types of plasminogen activators. The principle endogenous activator of plasminogen is the tissue-type plasminogen activator (tPA) ${ }^{[45]}$. Plasminogen activator inhibitor (PAI-1), which is produced by platelets, liver, and endothelium, on the other hand, is the major inhibitor of tPA. Generally, coagulation activation triggers a secondary activation of fibrinolysis that is rapidly shut off by the release of large amounts of PAI-1 ${ }^{[46]}$.

During acute dengue virus infection, coagulation parameters such as platelet counts, activated partial thromboplastin time (APTT) as well as fibrinolytic parameters of tPA and PAI-1 are altered. APTT is prolonged while tPA increases. Both coagulation and fibrinolysis are activated, and this activation is much more severe in DHF/DSS than in DF patients. After convalescence, rises in the PAI-1 level and platelet counts are concomitant with the decline in the tPA level and a return to normal of APTT. The tPA/PAI-1 ratio is higher in DHF/DSS than in DF patients. APTT prolongation and tPA/PAI-1 ratio increase in the acute stage of dengue virus infection correlate with disease severity and can be used as early indicators of $\mathrm{DHF} / \mathrm{DSS}^{[47]}$. APTT and prothrombin time are indicators of intrinsic and extrinsic pathways of coagulation, respectively. Only APTT, but not prothrombin time, is prolonged in dengue virus infection, suggesting that a defect occurs in the intrinsic pathway of coagulation. This can be caused by either downregulation of the synthesis of specific factors or by increased consumption of specific factors. Because mild hepatitis is found in dengue virus infection, an analysis of the linear correlation and regression between the levels of aspartate aminotransferase (AST)/alanine aminotransferase (ALT) and APTT shows a strong association between AST/ALT elevation and APTT prolongation in DHF patients. Dysfunction of the damaged liver might be responsible for the decreased synthesis of specific factors in the intrinsic pathway. Increased factor consumption as indicated by high levels of tPA is also associated with APTT prolongation, but in a less significant manner. Therefore, both decreased synthesis and increased consumption of coagulation factors are involved in the prolongation of APTT. 


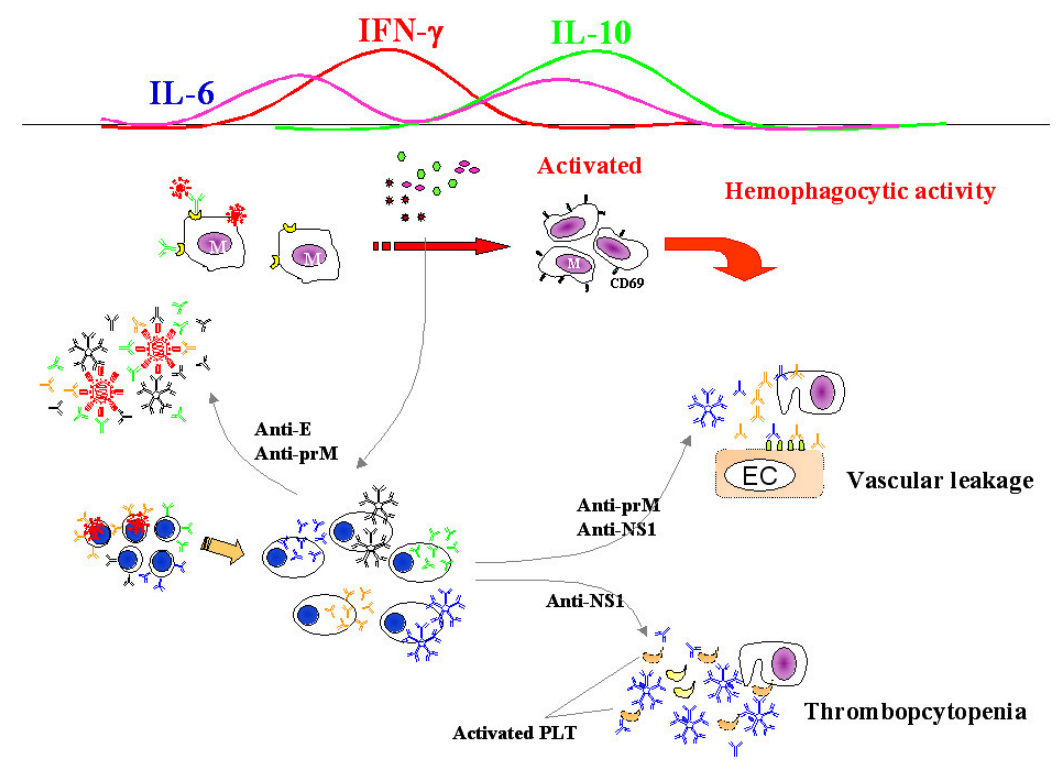

Fig. 1: Autoantibody-associated immunopathogenesis of dengue hemorrhagic fever. Dengue virus infection causes aberrant immune responses including CD4/CD8 ratio inversion, monocytosis, and atypical lymphocytosis, which not only delay virus clearance, but also trigger cytokine overproduction and autoantibodies to platelets and endothelial cells. These autoantibodies would subsequently initiate the dysfunctions of these cells. The IFN- $\gamma$ activated macrophages phagocytosize the autoantibody-coated platelets and endothelial cells, result in thrombocytopenia and endothelial cell damage. A transient hemophagocytic activity was induced post acute dengue virus infection

The hyperfibrinolysis in the acute stage of DHF/DSS is caused by increased production of tPA. Analysis of the linear correlation and regression shows a significant association between serum IL-6 and tPA in DHF, but not in DF. Dengue virus infection induces endothelial production of tPA as well as IL-6. The de novo synthesis of tPA is blocked by anti-IL-6 antibodies, indicating that tPA production by endothelial cells is IL-6 dependent ${ }^{[48]}$. Furthermore, antibodies against dengue virus $\mathrm{E}$ protein can bind to human plasminogen. It can either inhibit plasmin activity or enhance plasminogen activation ${ }^{[49]}$. Therefore, both coagulation and fibrinolysis are hyperactivated in the acute stage of dengue virus infection, and are counteracted by increased numbers of platelets and levels of PAI-1 in the convalescent stage. An unbalance between coagulation and fibrinolysis may cause hemorrhage in DHF/DSS.

Dengue virus can infect the liver and cause hepatitis. Elevated serum transaminase levels were found in dengue patients, and the degree of AST level elevation correlates with that of hemorrhage ${ }^{[50]}$. In dengue-viral hepatitis, the level of AST is higher than ALT with a ratio of around 1 1.5, while other types of virally induced hepatitis have more ALT than AST.
Using hepatoma cell lines, dengue virus can induce apoptosis as well as RANTES chemokine production via oxidative stress and NF- $\kappa B$ activation ${ }^{[51,52]}$. RANTES is preferentially induced by dengue virus but not enterovirus in liver cells. Patients with dengue virus infection have increased RANTES serum levels compared to those with other viral infections. RANTES is a chemokine capable of recruiting lymphocytes and NK cells to sites of inflammation. It is not clear whether the liver damage is a direct effect of virus replication or an indirect effect of RANTES-mediated inflammation. The balance between virus elimination and tissue damage might affect the severity of the disease. Since the liver is known to be the site for synthesis of most coagulation factors, reduced levels of coagulation factors are either the results of increased consumption or impaired synthesis. The latter is the likely consequence of liver injury. IL-6 can downregulate the synthesis of coagulation factor XII, the first factor to initiate the intrinsic pathway of coagulation $^{[53]}$. APTT prolongation in DHF patients caused by a deficiency of intrinsic pathway is probably due to impaired synthesis of coagulation factor XII in the liver. 
Immunopathogenesis of DHF: The characteristic features of DHF/DSS include capillary leakage, thrombocytopenia, and coagulopathy. Taking into consideration of several key observations such as: Dengue virus infection induces transient immune aberrant activation of $\mathrm{CD} 4 / \mathrm{CD} 8$ ratio inversion and cytokine overproduction. Dengue virus infection induces autoimmunity due to molecular mimicry. AntiNS1 or anti-prM antibodies can cross-react with platelets and endothelial cells. The binding to platelets causes platelet lysis in the presence of complement, whereas the binding to endothelial cells induces their NO-mediated apoptosis. Dengue virus infection activates both coagulation and fibrinolysis systems. Unbalances between coagulation and fibrinolysis induce hemorrhage in DHF/DSS. We propose a new immunopathogenesis of dengue virus infection-caused disease (Fig. 1).

Dengue virus infection causes intense immune activation. Aberrant immune responses such as cytokine overproduction and generation of autoantibody acting against platelets and endothelial cells occur after dengue virus infection. A molecular mimicry between platelets or endothelial cells with the NS-1 or prM of dengue virus would explain the cross-reactive of antiNS1 or anti-prM antibody to host cells, and participate in the attack of platelet and endothelial cells during the disease development. Dengue virus could cause severe hemophagocytic syndrome. High serum ferritin level, a macrophage activation marker in vivo, was found to be highly elevated in the dengue patients, suggesting that it is a common phenomenon in the dengue patients. This suggests that the monocytes or macrophages were activated by cytokines such as IFN- $\gamma$ during the dengue disease process after dengue virus infection. The activated macrophages would then achieve the phagocytosis of the autoantibody-coated platelet and thus contribute to the development of thrombocytopenia in DHF/DSS. The anti-NS1 and anti-prM cross-reactive antibody to platelets and endothelial cells provide an explanation for the target specificity and unique feature of thrombocytopenia and plasma leakage during the development of DHF/DSS. The macrophage activation might also be responsible for the sustained disease process, with a high fatality rate, as observed in the elderly dengue virus-infected patients.

\section{ACKNOWLEDGMENTS}

This study was supported by grant NHRI-CNCL9601S from the National Health Research Institute, and NSC95-3112-B006-004 from National Science Council, Republic of China.

\section{REFERENCES}

1. Bhamarapravati, N., P. Tuchinda and V. Boonyapaknavik, 1967. Pathology of Thailand haemorrhagic fever: A study of 100 autopsy cases. Ann. Trop. Med. Parasitol., 61: 500-510.

2. Burke, D.S., A. Nisalak, D.E. Johnson and R.M. Scott, 1988. A prospective study of dengue infection in Bangkok. Am. J. Trop. Med. Hyg., 38: 172-180.

3. Guzman, M.G. and G. Kouri, 2001. Dengue: An update. Lancet Infect. Dis., 2: 33-42.

4. Mackenzie, J.S., D.J. Gubler and L.R. Petersen, 2004. Emerging flaviviruses: The spread and resurgence of Japanese encephalitis, West Nile and dengue viruses. Nat. Med., 10: S98-S109.

5. Liu, J.W., B.S. Khor, C.H. Lee, I.K. Lee, R.F. Chen and K.D.Yang, 2003. Dengue hemorrhagic fever in Taiwan. Dengue Bullet., 27: 19-24.

6. Halstead, S.B., 1970. Observations related to pathogenesis of dengue hemorrhagic fever. VI. Hypotheses and discussion. Yale J. Biol. Med., 42: 350-362.

7. Halstead, S.B., 2003. Neutralization and antibodydependent enhancement of dengue viruses. Adv. Virus Res., 60: 421-467.

8. Kliks, S.C., A. Nisalak, W.E. Brandt, L. Wahl and D.S. Burke, 1989. Antibody-dependent enhancement of dengue virus growth in human monocytes as a risk factor for dengue hemorrhagic fever in infants. Am. J. Trop. Med. Hyg., 40: 444-451.

9. Kliks, S.C., S. Nimmanitya, A. Nisalak and D.S. Burke, 1988. Evidence that maternal dengue antibodies are important in the development of dengue hemorrhagic fever in infants. Am. J. Trop. Med. Hyg., 38: 411-419.

10. Rico-Hesse, R., L. Harrison, A. Nisalak, D.W. Vaugh, S. Kalayanarooj, S. Green, A.L. Rothman and F.A. Ennis, 1998. Molecular evolution of dengue type 2 virus in Thailand. Am. J. Trop. Med. Hyg., 58: 96-101.

11. Rico-Hesse, R., L. Harrison, R. Salas, D. Tovar, A. Nisalak, C. Ramos, J. Boshell, M. Rde Mesa, R. Nogueira and A. Travassos da Rosa, 1997. Origins of dengue 2 viruses associated with increased pathogenicity in the Americas. Virology, 230: 244-251.

12. Leitmeyer, K.C., D.W. Vaughn, D.M. Watts, R. Salas, I. Villalobos, C. deChacon Ramos and R. Rico-Hesse, 1999. Dengue virus structural difference that correlates with pathogenesis. J. Virol., 73: 4738-4747. 
13. Vaughn, D.W., S. Green, S. Kalayanarooj, B.L. Innis, S. Nimmannitya, S. Suntayakorn, T.P. Endy, B. Raengsakulrach, A.L.Rothman, F.A. Ennis and A. Nisalak, 2000. Dengue viremia titer, antibody response pattern, and virus serotype correlate with severity. J. Infect. Dis., 181: 2-9.

14. Vaughn, D.W., S. Green, S. Kalayanarooj, B.L. Innis, S. Nimmannitya, S. Suntayakorn, A.L. Rothman, F.A. Ennis and A. Nisalak, 1997. Dengue in the early febril phase: viremia and antibody responses. J. Infect. Dis., 176: 322-330.

15. Rothman, A.L., 2003. Immunology and Immunopathogenesis of Dengue Disease. In: Maramorosch, K., A.L. Shatkin, F.A. Murphy, T.J. Chambers and T.P. Monath (Eds.). Flaviviruses: Pathoggnesis and Immunity, Academic Press Inc., San Diego, CA., 60: 397-419

16. Rothman, A.L., 2004. Dengue: Ddefining protective versus pathologic immunity. J. Clin. Invest., 113: 946-951.

17. Lei, H.Y., T.M. Yeh, H.S. Liu, Y.S. Lin, S.H. Chen and C.C. Liu, 2001. Immunopathogensis of dengue virus infection. J. Biomed. Sci., 8: 377-388.

18. Diamond, M.S., D. Edgil, G. Roberts, B. Lu and E. Harris, 2000. Infection of human cells by dengue virus is modulated by different cell types and viral strains. J. Virol., 74: 7814-7823.

19. Liu, C.C., K.J. Huang, Y.S. Lin, T.M. Yeh, H.S. Liu and H.Y. Lei, 2002. Transient CD4/CD8 ratio inversion and immune deviation during dengue virus infection. J. Med. Virol., 68: 241-252.

20. Wu, S.J.L., G. Grouard-Vogel, W. Sun, J.R. Mascola, E. Brachtel, R. Putvatana, M.K. Louder, L. Filgueira, M.A. Marovich, H.K. Wong, A. Blauvelt, G.S. Murphy, M.L. Robb, B.L. Innes, D.L. Birx, C.G. Hayes and S.S. Frankel, 2000. Human skin Langerhans cells are targets of dengue virus infection. Nat. Med., 6: 816-820.

21. Ho, L.J., J.J. Wang, M.F. Shaio, C.L. Kao, D.M Chang and J.H. Lai, 2001. Infection of human dendritic cells by dengue virus causes cell maturation and cytokine production. J. Immunol., 166: 1499-1506.

22. Pacsa, A.S., R. Agarwal, E.A. Elbishbishi, U.C. Chaturvedi, R. Nagar and A.S. Mustafa, 2000. Role of interleukin-12 in patients with dengue hemorrhagic fever. FEMS Immunol. Med. Microbiol., 28: 151-155.

23. Lin, Y.W., K.J. Wang, H.Y. Lei, Y.S Lin, T.M. Yeh, H.S. Liu, C.C. Liu and S.H. Chen, 2002. Virus replication and cytokine production in dengue virus-infected human B lymphocytes. J. Virol., 76: 12242-12249.

24. King, A.D., A. Nisalak, S. Kalayanrooj, K.S.A. Myint, K. Pattanapanyasat, S. Nimmannitya and B.L. Innis, 1999. B cells are the principal circulating mononuclear cells infected by dengue virus. Southeast Asian J. Trop. Med. Public Health, 30: 718-728.

25. Kurane, I., B.L. Innis, S. Nimmannitya, A. Nisalak, A. Meager, J. Janus and F.A. Ennis, 1991. Activation of T-lymphocytes in dengue virus infection. High levels of soluble interleukin 2 receptor, soluble CD4, soluble CD8, interleukin 2, and interferon-gamma in sera of children with dengue. J. Clin. Invest., 88:1473-1480.

26. Kurane, I., Innis, B.L., Nimmannitya, S., Nisalak, A., Meager, A., Ennis and F.A, 1993. High levels of interferon alpha in the sera of children with dengue virus infection. Am. J. Trop. Med. Hyg., 48: 222-229.

27. Green, S., D.W. Vaughn, S. Kalayanarooj, S. Nimmannitya, S. Suntayakorn, A. Nisalak, A.L. Rothman and F. A. Ennis, 1999. Elevated plasma interleukin-10 levels in acute dengue correlate with disease severity. J. Med. Virol., 59: 329334.

28. Hung, N.T., H.Y. Lei, N.T. Lan, Y.S. Lin, K.J. Huang, L.B. Lien, C.F. Lin, T.M. Yeh, D.Q. Ha, V. T. Q. Huong, L.C. Chen, J.H. Huang, L.T. My, C.C. Liu and S.B. Halstead, 2004. Dengue hemorrhagic fever in infants: A study on clinical and cytokine profiles. J. Infect. Dis., 189: 221-232.

29. Rothman, A.L. and F.A. Ennis, 1999. Immunopathogenesis of dengue hemorrhagic fever. Virology, 257: 1-6.

30. La Russa, V.F. and B.L. Innis, 1995. Mechanisms of dengue virus-induced bone marrow suppression. Baillieres Clin. Haem., 8: 249-270.

31. Wang, S., R. He, J. Patarapotikul, B.L. Innis and R. Anderson, 1995. Antibody-enhanced binding of dengue-2 virus to human platelets. Virology, 213: 254-257.

32. Heegaard, E.D., S. Rosthoj, B.L. Petersen, S. Nielsen, F. Karup Pedersen and A. Hornsleth, 1999. Role of parvovirus B19 infection in childhood idiopathic thrombocytopenic purpura. Acta Paediatr, 88: 614-617.

33. Yoto, Y., T. Kudoh, N. Suzuki, S. Katoh, Y. Matsunaga and S. Chiba, 1993. Thrombocytopenia induced by human parvovirus B19 infections. Eur. J. Haematol., 50: 255-257.

34. Lin, C,F., H.Y. Lei, C.C. Liu, H.S. Liu, T.M. Yeh, S.T. Wang, T.I. Yang, F.C. Sheu, C.F. Kuo and Y.S. Lin, 2001. Generation of IgM anti-platelet 
autoantibody in dengue patients. J. Med. Virol., 63: 143-149.

35. Huang, K.J., S.Y. J. Li, S.C. Chen, H.S. Liu, Y.S. Lin, T.M. Yeh, C.C. Liu and H.Y. Lei, 2000. Manifestation of thrombocytopenia in dengue-2virus-infected mice. J. Gen. Virol., 81: 2177-2182.

36. Lin, C.F., S.W. Wan, H.J. Cheng, H.Y. Lei and Y.S. Lin, 2006. Autoimmune pathogenesis in dengue virus infection. Viral Immunol, 19: 127-132.

37. Lin, C.F., H.Y. Lei, A.L. Shiau, C.C. Liu, H.S. Liu, T.M. Yeh, S.H. Chen and Y.S. Lin, 2003. Anitbodies from dengue patient sera cross-react with endothelial cells and induce damage. J. Med. Virol., 69: 82-90.

38. Avirutnan, P., P. Malasit, B. Seliger, S. Bhakdi and M. Husmann, 1998. Dengue virus infection of human endothelial cells leads to chemokine production, complement activation, and apoptosis. J. Immunol., 161: 6338-6346.

39. Huang, Y.H., H.Y. Lei, H.S. Liu, Y.S. Lin, C.C. Liu and T.M. Yeh, 2000. Dengue virus infects human endothelial cells and induces IL-6 and IL-8 production. Am. J. Trop. Med. Hyg., 63: 71-75.

40. Lin, C.F., H.Y. Lei, A.L. Shiau, H.S. Liu, T.M. Yeh, S.H. Chen, C.C. Liu, S.C. Chiu and Y.S. Lin, 2002. Endothelial cell apoptosis induced by antibodies against dengue virus nonstructural protein 1 via production of nitric oxide. J. Immunol., 169: 657-664.

41. Lin, C.F., H.Y. Lei, A.L. Shiau, H.S. Liu, T.M. Yeh, S.H. Chen, C.C. Liu, S.C. Chiu and Y.S. Lin, 2002. Endothelial cell apoptosis induced by antibodies against dengue virus nonstructural protein 1 via production of NO. J. Immunol., 169: 657-664.

42. Lin, Y.S., C.F. Lin, H.Y. Lei, H.S. Liu, T.M. Yeh, S.H. Chen and C.C. Liu, 2004. Antibody-mediated endothelial cell damage via nitric oxide. Curr. Pharmaceut. Design, 10: 213-221.

43. Huang, K.J., Y.C. Yang, Y.S. Lin, J.H. Huang, H.S. Liu, T.M. Yeh, S.H. Chen, C.C. Liu and H.Y. Lei, 2006. The dual-specific binding of dengue virus and target cells for the antibodydependent enhancement of dengue virus infection. J. Immunol., 176: 2825-2832.

44. Huang, Y.H., 2000. Study on the mechanism of hemorrhage induced by dengue virus infection. Ph.D. Thesis, Tainan, Taiwan: National Cheng Kung University.
45. Bachmann, F. and E.K.O. Kruithof, 1984. Tissue plasminogen activator: Chemical and physiological aspects. Semin. Thromb. Hemost., 10: 6-17.

46. Van Gorp, E.C.M., C. Shuarti, H. ten Cate, W.M.V. Dolmans, J.W. M. van der Meer, J.W. ten Cate and D.P.M. Brandjes, 1999. Review: Infectious diseases and coagulation disorders. J. Infect. Dis., 180:176-186.

47. Huang, Y.H., C.C. Liu, S.T. Wang, H.Y. Lei, H.S. Liu, Y.S. Lin, H.L. Wu and T.M. Yeh, 2001. Activation of coagulation and fibrinolysis during dengue virus infection. J. Med. Virol., 63: 247-251.

48. Huang, Y.H., H.Y. Lei, H.S. Liu, Y.S. Lin, S.H. Chen, C.C. Liu and T.M. Yeh, 2003. Tissue plasminogen activator induced by dengue virus infection of human endothelial cells. J. Med. Virol., 70: 610-616.

49. Huang, Y.H., B.I. Chang, H.Y. Lei, H.S. Liu, C.C. Liu, H.L. Wu and T.M. Yeh, 1997. Antibodies against dengue virus $\mathrm{E}$ protein peptide bind to human plasminogen and inhibit plasmin activity. Clin. Exp. Immunol., 110: 35-40.

50. Kalayanarooj, S., D.W. Vaughn, S. Nimmannitya, S. Green, S. Suntayakorn, N. Kunentrasai, W. Viramitrachai, S. Ratanachueke, S. Kiatpolpoj, B.L. Innis, A.L. Rothman, A. Nisalak and F.A. Ennis, 1997. Early clinical and laboratory indicators of acute dengue illness. J. Infect. Dis., 176: 313-321.

51. Lin, Y.L., C.C. Liu, H.Y. Lei, T.M. Yeh, Y.S. Lin, R.M.Y. Chen and H.S. Liu, 2000. Infection of five liver cell lines by dengue-2 virus. J. Med. Virol., 60: 425-431.

52. Lin, Y.L., C.C. Liu, J.I. Chuang, H.Y. Lei, T.M. Yeh, Y.S. Lin, Y.H. Huang and H.S. Liu, 2000. Involvement of oxidative stress, NF-IL-6 and RANTES expression in dengue-2 virus infected human liver cells. Virology, 276: 114-126.

53. Citarella, F., A. Felici, M. Brouwer, J. Wagstaff, A. Fantoni and C.E. Hack, 1997. Interleukin-6 downregulates factor XII production by human hepatoma cell line (HepG2). Blood, 90: 1501-1507. 